\title{
An extreme creA mutation in Aspergillus nidulans has severe effects on D-glucose utilization
}

\author{
Peter van der Veen, George J. G. Ruijter and Jaap Visser
}

Author for correspondence: Jaap Visser. Tel: +318370 84439. Fax: +31837084011.

e-mail: office@ algemeen.mgim.wau.nl

Wageningen Agricultural University, Section of Molecular Genetics of Industrial Microorganisms, Dreyenlaan 2, $6703 \mathrm{HA}$

Wageningen, The Netherlands

\begin{abstract}
Aspergillus nidulans wild-type and the extreme carbon catabolite derepressed mutant cre $A^{d}-30$ were characterized with respect to enzyme activities, metabolite concentrations and polyol pools all related to glycolysis, after growth on 0 -glucose. In the cre $A^{d}-30$ strain the enzymes hexokinase and fructose-6-phosphate reductase showed a two- and threefold increase in activity, respectively, whereas phosphofructokinase and pyruvate kinase activity decreased two- and threefold, respectively, in comparison with the wild-type strain. The most notable changes in metabolite concentrations were that fructose 2,6-bisphosphate and fructose 1,6-bisphosphate showed a 2.5fold increase, whereas both pyruvate and citrate decreased in the cre $A^{d}-30$. Striking differences were found for the polyol concentrations measured for the two strains tested. Intracellular glycerol and arabitol concentrations were 10-fold higher and erythritol fivefold higher in creAd $^{d}-30$, whereas intracellular trehalose and mannitol were both decreased. The total internal polyol concentration appears to be constant at $\sim 700 \mu \mathrm{mol}\left(\mathrm{g}\right.$ dry wt) ${ }^{-1}$. All polyols were also detected in high amounts in the culture filtrate of the cre $A^{d-30}$ mutant strain but no extracellular trehalose was found. The overall production of polyols in this strain was therefore much higher than in the wild-type. The high level of polyols produced and the changes in metabolite concentrations in the cre $^{d}-30$ strain suggest that the differences in enzyme activities result in an altered flow through glycolysis leading to a more rapid formation of polyols which are subsequently secreted.
\end{abstract}

Keywords: Aspergillus nidulans, carbon catabolite repression, glycolytic enzyme/metabolite levels, metabolic flux, energy charge

\section{INTRODUCTION}

Carbon catabolite repression provides microbial cells with a means to adapt their metabolism so that favoured carbon sources such as $\mathrm{D}$-glucose, $\mathrm{D}$-fructose or $\mathrm{D}$-xylose are utilized preferentially in the presence of other less preferred carbon sources. Mutations in the hyphal fungus Aspergillus nidulans resulting in altered responses to carbon catabolite repression have been known for approximately

Abbreviations: DHA, dihydroxyacetone; DHAP, dihydroxyacetonephosphate; F6P, fructose 6-phosphate; F1,6bP, fructose 1,6-bisphosphate; F2,6bP, fructose 2,6-bisphosphate; G1P, glucose 1-phosphate; G3P, glycerol 3-phosphate; G6P, glucose 6-phosphate; M1P, mannitol 1-phosphate; PEP, phosphoenolpyruvate; 1,3bPG, 1,3-bisphosphoglycerate; 2PG, 2phosphoglycerate; 3PG, 3-phosphoglycerate; 6PG, 6-phospho-gluconolactone; T6P, trehalose 6-phosphate; UDPG, UDP-glucose. two decades (Arst \& Cove, 1973; Bailey \& Arst, 1975). The strategies devised by these authors to obtain carbon catabolite derepressed mutations were based on the selection of pseudorevertants using parental strains with a specific genetic background. Mutants able to grow on a mixture of glucose and L-proline were, for instance, selected from an ammonium-requiring are $A$-defective parental strain. Similarly, carbon catabolite derepressed mutants were obtained from a glucose non-utilizing $p d h A$ mutant which itself was unable to utilize ethanol in the presence of glucose due to carbon catabolite repression of alcohol- and aldehyde dehydrogenases.

The most extreme cre $A$ mutant isolated is the $\operatorname{cre} A^{d}-30$. This mutant was selected as a spontaneous mutation showing resistance to the toxicity of $\mathrm{D}$-mannitol in a fr $A$ 1 background (Arst et al., 1990). Of the cre $A$ gene thus 
identified, a number of mutant alleles have been selected which are recessive to wild-type and which typically display non-hierarchical heterogeneity of mutant phenotypes (Arst \& Bailey, 1977; van der Veen et al., 1994). The CREA protein was postulated to be a negatively acting regulatory protein directly involved in regulating gene expression (Arst \& Bailey, 1977; Arst \& MacDonald, 1975). This was confirmed by Dowzer \& Kelly (1989, 1991) who cloned $\operatorname{cre} A$ and found it to encode a 'zinc finger' DNA-binding protein of the $\mathrm{Cys}_{2} \mathrm{His}_{2}$ type. A fusion protein containing the 'zinc finger' domain of $A$. nidulans CREA showed sequence-specific binding to promoters of genes which are under carbon catabolite repression control, such as those of the ethanol regulon (Kulmburg et al., 1993) or the proline gene cluster (Cubero \& Scazzocchio, 1994). Although the direct role of the CREA protein as a repressor of gene expression has now been confirmed (Cubero \& Scazzocchio, 1994; Mathieu \& Felenbok, 1994), the physiological consequences of this extreme $\operatorname{cre} A^{d}$-30 mutation have not yet been investigated. We therefore decided to investigate the physiological changes occurring upon growth on $\mathrm{D}$ glucose in a mutant $\operatorname{cre} A$ strain compared to wild-type $A$. nidulans. In this paper we present data on enzyme activities and metabolite levels related to glycolysis and energy metabolism. In addition, intracellular and extracellular polyol concentrations were studied.

\section{METHODS}

Materials. All chemicals used were of analytical quality and obtained from Merck. All enzymes and substrates were obtained from Boehringer Mannheim except for fructose 2,6-bisphosphate (F2,6bP) and pyrophosphate-dependent fructose-6phosphate kinase which were obtained from Sigma.

Strains and growth conditions. A. nidulans strain WG096 (yA2 paba $A 1)$ was used as the wild-type strain. The $A$. nidulans carbon catabolite derepressed mutant strain used was cre $A^{d}$-30 (biA1) (Arst et al., 1990). The strains were grown on minimal medium as previously described by Pontecorvo et al. (1953). $p$ Aminobenzoic acid was added to a final concentration of $2 \mathrm{mg}$ $\mathrm{I}^{-1}$ and biotin to a final concentration of $4 \mu \mathrm{g}^{-1}$ to the cultures. Both strains were grown at $37^{\circ} \mathrm{C}$ for $16 \mathrm{~h}$ in $250-300 \mathrm{ml}$ minimal medium, $0.05 \%(\mathrm{w} / \mathrm{v})$ yeast extract and $1.5 \%(\mathrm{w} / \mathrm{v}) \mathrm{D}-$ glucose in 11 Erlenmeyer flasks using a New Brunswick orbital shaker at 250 r.p.m. All cultures were inoculated to $1 \times 10^{6}$ spores $\mathrm{ml}^{-1}$.

Preparation of cell-free extracts and protein determination. The preparation of cell-free extracts from mycelium was carried out as described by Witteveen et al. (1989). Protein concentrations were measured using the Sigma BCA method as described by van der Veen et al. (1991).

Determinations of metabolites. The method to extract metabolites from mycelium as developed by G. J. G. Ruijter \& J. Visser (unpublished results) for Aspergillus niger was used. It is a modification of the method described by de Koning \& van Dam (1992). Glycolytic metabolites were assayed enzymically according to the methods of Bergmeyer (1985a, b). F2,6bP was determined by a kinetic method by measuring the rate of $\mathrm{PP}_{1^{-}}$ dependent phosphofructokinase as described by Van Schaftingen et al. (1982). All analyses were performed on a Cobas Bio Autoanalyser (Roche) which was connected to an
MS-DOS computer for datalogging. Volumes in published methods were adapted to the working volume of the apparatus $(0 \cdot 25 \mathrm{ml})$. Analyses were performed at $30^{\circ} \mathrm{C}$.

Enzyme determinations. All enzyme determinations were carried out using an Aminco DW-2 UV/VIS double beam spectrophotometer (Silver Spring) with an Atari computer for datalogging and calculations. The enzyme activities were measured at $37^{\circ} \mathrm{C}$ essentially as described by Bergmeyer (1985a, $1985 \mathrm{~b}$ ) but using $50 \mathrm{mM}$ PIPES buffer, $\mathrm{pH} 7 \cdot 5$, containing $100 \mathrm{mM} \mathrm{KCl}$ and $5 \mathrm{mM} \mathrm{MgCl}_{2}$ in all cases.

Polyol extraction and determination. The intracellular polyol determinations were carried out as described by Witteveen et al. (1993). The extracellular polyol determinations were done as follows. Just before harvesting of the mycelia, a sample was taken from the culture filtrate and spun down for $5 \mathrm{~min}$ in an Eppendorf centrifuge to remove mycelia and debris. The sample was diluted fivefold with deionized water from a Millipore filtration unit, and the polyol concentrations were determined by high performance anion-exchange chromatography (HPAEC) on a Dionex system with a CarboPac MA1 column using isocratic elution with $0.48 \mathrm{M} \mathrm{NaOH}$ as described by Witteveen et al. (1993).

\section{RESULTS}

\section{Enzyme activities}

An analysis of glycolytic enzyme patterns and metabolite levels in the $A$. nidulans WG096 wild-type strain and a genotypically defined extreme derepressed $\operatorname{cre} A^{d}$-30 mutant was initiated to investigate the physiological consequences of this mutation. Fig. 1 shows an overview of glycolysis with some of its branches.

After $16 \mathrm{~h}$ of growth, samples were taken from the mycelia and the culture broth for further analysis. The $\mathrm{D}^{-}$ glucose concentration was found to be high enough to exert full carbon catabolite repression (wild-type, $35 \mathrm{mM}$; $\operatorname{cre} A^{d}-30,40 \mathrm{mM}$ ). The total biomass produced in these cultures was 0.823 and $0.725 \mathrm{~g}$ dry weight per $250 \mathrm{ml}$ culture for wild-type and $\operatorname{cre} A^{d}-30$, respectively.

Following growth of the wild-type and $\operatorname{cre} A^{d}$-30 strains on D-glucose we found differences in the activity of several glycolytic enzymes (Table 1). The first notable difference was the increased hexokinase activity in $\operatorname{cre} A^{d}$ 30. The glucose 6-phosphate (G6P) pool formed by hexokinase is distributed to supply the glycolytic and pentose phosphate pathways as well as to synthesize UDP-glucose (UDPG). Moreover, G6P is required for the synthesis of trehalose. There were no significant changes found between the wild-type and the cre $A^{d}$-30 strain for any of the enzymes involved in G6P metabolism. At the level of fructose 6-phosphate (F6P) metabolism, however, two significant changes were found (cf. Fig. 1). Phosphofructokinase, one of the key regulatory enzymes in glycolysis in many organisms, was decreased in the cre $A^{a}-30$ mutant strain. Another enzyme that converts F6P into mannitol 1-phosphate (M1P) is fructose-6phosphate reductase. The M1P formed is subsequently converted into mannitol and is initially stored. Fructose6-phosphate reductase levels were threefold higher in the cre $A^{d}-30$ mutant. Interestingly, this was the opposite 


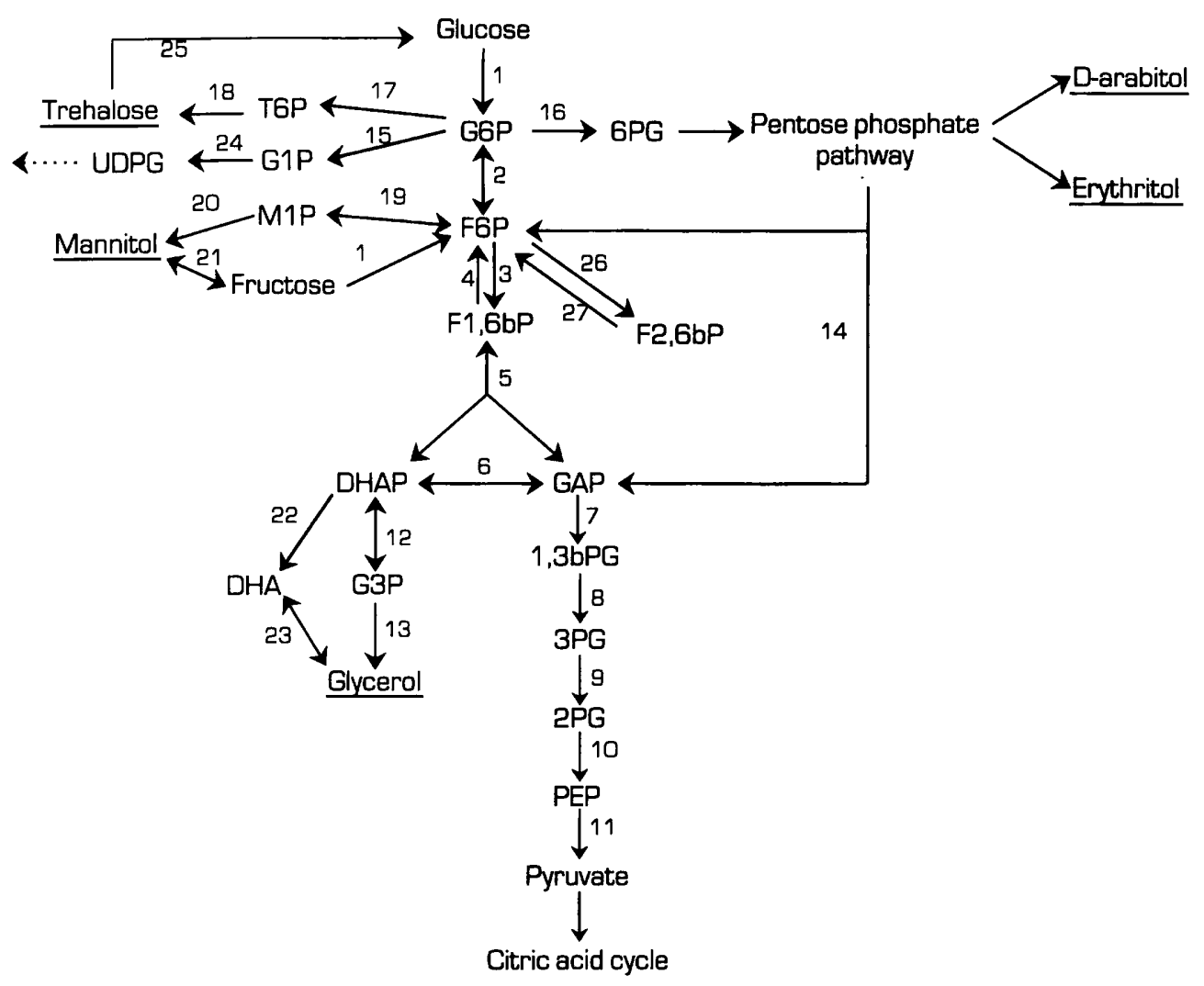

Fig. 1. Part of the hexose monophosphate pathway and metabolic routes involved in polyol synthesis. Enzymes: 1, hexokinase; 2 , phosphoglucose isomerase; 3, phosphofructokinase; 4, fructose-1,6-bisphosphatase; 5, aldolase; 6 , triose phosphate isomerase; 7, glyceraldehyde-3-phosphate dehydrogenase; 8, phosphoglycerate kinase; 9, phosphoglyceromutase; 10 , enolase; 11, pyruvate kinase; 12, glycerol-3-phosphate dehydrogenase; 13, glycerol-3phosphate phosphatase; 14 , transketolase; 15, phosphoglucomutase; 16, glucose-6-phosphate dehydrogenase; 17, trehalose-6-phosphate synthase; 18, trehalose-6-phosphate phosphatase; 19, fructose-6-phosphate reductase; 20, mannitol-1-phosphate phosphatase; 21, mannitol dehydrogenase; 22, dihydroxyacetonephosphate phosphatase; 23, glycerol dehydrogenase; 24, UDP-glucose pyrophosphorylase; 25, trehalase; 26, fructose-6-phosphate-2-kinase; 27, fructose-2,6-bisphosphatase.

Table 1. Activities of various intracellular enzymes involved in glucose metabolism in extracts of $A$. nidulans wild-type and $c r e A^{d}-30$ mutant strains

Data are the means of two independent experiments. Strains were grown for $16 \mathrm{~h}$ on $1.5 \%(\mathrm{w} / \mathrm{v}) \mathrm{D}$-glucose at $37^{\circ} \mathrm{C}$. Activities are expressed in $\mathrm{U}(\mathrm{mg} \text { protein })^{-1} \pm \mathrm{SD}$.

\begin{tabular}{|lcc|}
\hline Enzyme & Wild-type & cre $\boldsymbol{A}^{\boldsymbol{d}}$-30 \\
\hline Hexokinase & $0 \cdot 28 \pm 0.02$ & $0.46 \pm 0.02$ \\
Glucose-6-P dehydrogenase & $2 \cdot 18 \pm 0.05$ & $1 \cdot 99 \pm 0.01$ \\
Phosphoglucomutase & $1 \cdot 22 \pm 0.04$ & $1 \cdot 56 \pm 0.05$ \\
Phosphoglucose isomerase & $2 \cdot 24 \pm 0 \cdot 13$ & $1 \cdot 95 \pm 0.09$ \\
Fructose-6-P reductase & $0 \cdot 53 \pm 0.02$ & $1 \cdot 86 \pm 0.03$ \\
Phosphofructokinase & $1 \cdot 18 \pm 0.02$ & $0 \cdot 46 \pm 0.02$ \\
Fructose-1,6-bisphosphatase & $0.06 \pm 0.005$ & $0 \cdot 14 \pm 0.01$ \\
Aldolase & $1 \cdot 86 \pm 0 \cdot 14$ & $1 \cdot 83 \pm 0 \cdot 14$ \\
Triose phosphate isomerase & $18.26 \pm 0.32$ & $16.83 \pm 0.51$ \\
Pyruvate kinase & $1.50 \pm 0 \cdot 1$ & $0.56 \pm 0.1$ \\
Citrate synthase & $1 \cdot 08 \pm 0.01$ & $0.64 \pm 0.01$ \\
\hline
\end{tabular}

effect to that observed for phosphofructokinase. Another important enzyme activity in this connection is, of course, fructose-1,6-bisphosphatase which catalyses the reverse reaction of phosphofructokinase (cf. Fig. 1). This activity was low in both strains and although somewhat higher in the cre $A^{d}$ - 30 mutant, it is not likely that this increase can account for the significant physiological changes in the $\operatorname{cre} A^{d}-30$ strain. The other glycolytic enzyme activities measured, viz. triose phosphate isomerase and aldolase, were high and not altered in the $\operatorname{cre} A^{d}$-30 strain.

As for the other two enzymes measured, pyruvate kinase activity was threefold lower and citrate synthase activity was $1 \cdot 7$-fold lower in the $\operatorname{cre} A^{d}$-30 mutant compared with the wild-type.

\section{Metabolite levels}

Data on enzyme levels alone provides only limited information as the enzyme activities in vitro define only the capacity of the particular enzymic steps in the pathway. By measuring metabolite levels in addition, information is 
Table 2. Metabolite determinations in mycelia of $A$. nidulans wild-type and $c r e A^{d}-30$ strains

Data are the means of three independent experiments. Concentrations are expressed in $\mu \mathrm{mol}$ ( $\mathrm{g}$ dry $\mathrm{wt})^{-1} \pm \mathrm{sD}$.

\begin{tabular}{|lcc|}
\hline Metabolite & Wild-type & cre $\boldsymbol{A}^{\boldsymbol{d}}$-30 \\
\hline Glucose 6-phosphate & $1 \cdot 23 \pm 0 \cdot 34$ & $1 \cdot 02 \pm 0 \cdot 21$ \\
Fructose 6-phosphate & $0 \cdot 20 \pm 0 \cdot 05$ & $0 \cdot 22 \pm 0 \cdot 04$ \\
Fructose 1,6-bisphosphate & $0 \cdot 34 \pm 0 \cdot 04$ & $0 \cdot 73 \pm 0 \cdot 08$ \\
Fructose 2,6-bisphosphate & $0 \cdot 00135 \pm 0 \cdot 00014$ & $0 \cdot 00275 \pm 0 \cdot 00021$ \\
3-Phosphoglycerate & $0 \cdot 08 \pm 0 \cdot 03$ & $0 \cdot 12 \pm 0 \cdot 01$ \\
2-Phosphoglycerate & $0 \cdot 07 \pm 0 \cdot 03$ & $0 \cdot 12 \pm 0 \cdot 04$ \\
Phosphoenolpyruvate & $0 \cdot 18 \pm 0 \cdot 11$ & $0 \cdot 29 \pm 0 \cdot 15$ \\
Pyruvate & $3 \cdot 82 \pm 0 \cdot 22$ & $1 \cdot 49 \pm 0 \cdot 48$ \\
Citrate & $7 \cdot 04 \pm 0 \cdot 67$ & $2 \cdot 42 \pm 0 \cdot 23$ \\
ATP & $1 \cdot 37 \pm 0 \cdot 28$ & $1 \cdot 49 \pm 0 \cdot 44$ \\
ADP & $1 \cdot 88 \pm 0 \cdot 43$ & $1 \cdot 66 \pm 0.38$ \\
AMP & $0 \cdot 75 \pm 0 \cdot 12$ & $0 \cdot 67 \pm 0 \cdot 18$ \\
\hline
\end{tabular}

obtained about the actual cellular concentrations of the various reactants, viz. substrates, products and effectors from which actual metabolic rates can be calculated. The intracellular metabolite levels are presented in Table 2. The levels of G6P and F6P were quite similar in wild-type and in the $\operatorname{cre} A^{d}-30$ strain. However, in between the phosphofructokinase and pyruvate kinase reaction steps elevated levels of the glycolytic intermediates F1,6bP, 2phosphoglycerate (2PG), 3-phosphoglycerate (3PG) and phosphoenolpyruvate (PEP) were found. In contrast, levels of pyruvate and citrate, which depend on pyruvate kinase activity, were lower in the $\operatorname{cre} A^{d}$ - 30 mutant.

Another striking change in $\operatorname{cre} A^{d}-30$ was seen at the level of $\mathrm{F} 2,6 \mathrm{bP}$ which is a potent activator of phosphofructokinase. Similarly to F1,6bP, a two- to threefold increase was found in the mutant. To determine whether the metabolic changes observed in glycolysis in the cre $A^{d}$ 30 mutant strain would also have consequences for the energy state of the cells, the levels of the total adenine nucleotide pools present were measured. The values for the energy charge calculated on the basis of these measurements fluctuate between 0.5 and 0.6 and do not suggest drastic changes.

\section{Polyol pools}

A. nidulans forms several polyols from D-glucose. Mannitol and glycerol are derived from glycolytic intermediates, whereas arabitol and erythritol arise from pentose phosphate pathway intermediates. Initially the polyols accumulate inside the fungal cell, but finally they are also excreted. Re-utilization of these polyols requires the induction of specific enzymes which are usually under carbon catabolite repression control. We therefore compared wild-type $A$. nidulans and the $\operatorname{cre} A^{d}$ - 30 mutant with respect to the intracellular and extracellular polyols formed upon growth on D-glucose (Table 3). The major polyol present in the wild-type upon growth on D-glucose was mannitol. In addition, some trehalose and erythritol
Table 3. Intracellular and extracellular polyol concentrations in $A$. nidulans wild-type and $c r e A^{d}-30$ mutant strains

Data are the means of two independent experiments. Strains were grown for $16 \mathrm{~h}$ on $1.5 \%(\mathrm{w} / \mathrm{v}) \mathrm{D}$-glucose at $37^{\circ} \mathrm{C}$. Intracellular concentrations are expressed in $\mu \mathrm{mol}(\mathrm{g} \mathrm{dry} \mathrm{wt})^{-1}$; extracellular concentrations are expressed in $\mathrm{mM}$.

\begin{tabular}{|c|c|c|c|c|}
\hline \multirow[t]{2}{*}{ Polyol } & \multicolumn{2}{|c|}{ Intracellular } & \multicolumn{2}{|c|}{ Extracellular } \\
\hline & Wild-type & cre $A^{d}-30$ & Wild-type & $\operatorname{cre} A^{d}-30$ \\
\hline Mannitol & $623 \cdot 1$ & $469 \cdot 4$ & 0.61 & $2 \cdot 71$ \\
\hline Trehalose & $32 \cdot 7$ & $2 \cdot 3$ & 0 & 0 \\
\hline Erythritol & $26 \cdot 9$ & $139 \cdot 7$ & $0 \cdot 14$ & $2 \cdot 36$ \\
\hline Glycerol & $7 \cdot 1$ & $65 \cdot 6$ & $0 \cdot 18$ & 0.53 \\
\hline Arabitol & $2 \cdot 2$ & $28 \cdot 2$ & 0 & $0 \cdot 16$ \\
\hline Total & 692 & 705 & 0.93 & $5 \cdot 76$ \\
\hline
\end{tabular}

accumulated whereas only small amounts of glycerol and arabitol were found. In the $\operatorname{cre} A^{d}$-30 strain this pattern changed completely. Both mannitol and trehalose levels were much lower in cre $A^{d}$-30, whereas the concentrations of arabitol (10-fold) and erythritol (15-fold) were greatly increased. The total intracellular concentration of these polyols including trehalose was found to be similar in

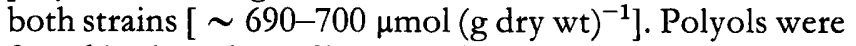
found in the culture filtrates of both the wild-type and the cre $A^{d}-30$ strain but no trehalose could be detected in either, whereas arabitol was only present in the culture filtrate of the cre $A^{d}-30$ mutant. Extracellular polyols were present in much higher concentrations in the medium of the cre $A^{d}$-30 strain (cf. Table 3).

Table 4 shows the total amounts of the various polyols produced by the wild-type and the $\operatorname{cre} A^{d}-30$ mutant strains. Trehalose was the only polyol produced more in the wild-type (25-fold), whereas the other polyol levels 
Table 4. Total amount of intracellular and extracellular polyols produced by $A$. nidulans wild-type and $c r e A^{d}-30$ mutant strain

Concentrations are expressed in $\mu \mathrm{mol}$ per $250 \mathrm{ml}$ culture.

\begin{tabular}{|lrr|}
\hline Polyol & Wild-type & ${\text { cre } \boldsymbol{A}^{\boldsymbol{d}} \text {-30 }}^{\text {-30 }}$ \\
\hline Mannitol & 666 & 1063 \\
Trehalose & 27 & 2 \\
Erythritol & 57 & 705 \\
Glycerol & 51 & 186 \\
Arabitol & 2 & 64 \\
Total & 803 & 2020 \\
\hline
\end{tabular}

were higher in the $\operatorname{cre} A^{d}$-30 strain (glycerol, threefold; erythritol, 12-fold; arabitol, 28-fold; mannitol, 1.5-fold). Also, the total amount of the polyols formed in the cre $A^{a}$ 30 mutant was almost threefold higher than in the wildtype strain.

\section{DISCUSSION}

The enzymic data obtained suggest that a number of enzymes in D-glucose metabolism are, directly or indirectly, influenced by the CREA protein, namely hexokinase, fructose-6-phosphate reductase, phosphofructokinase, pyruvate kinase and citrate synthase.

Hexokinase, the first enzyme in glycolysis (Fig. 1), was elevated in the cre $A^{d}$ - 30 strain (Table 1) whereas phosphofructokinase was lowered. However, these changes did not alter concentrations of G6P and F6P (Table 2), suggesting that overflow may occur via routes branching off from glycolysis (Fig. 1). Two possibilities are the conversion of F6P to M1P by fructose-6-phosphate reductase which leads to the formation of mannitol and the conversion of G6P to 6-phospho-gluconolactone (6PG) by glucose-6-phosphate dehydrogenase which can lead, via the pentose phosphate pathway, to arabitol and erythritol formation. Fructose-6-phosphate reductase (Table 1) was greatly increased in the cre $A^{d}-30$ strain. Although the internal mannitol concentration (Table 3 ) is lower than in the wild-type, the total amount of mannitol formed by the cre $A^{d}$-30 mutant is higher (Table 4). Furthermore, high internal and external concentrations of arabitol and erythritol (Table 3) were found in the $\operatorname{cre} A^{d_{-}}$ 30 strain, and these high internal concentrations may have some toxic effect in the cre $A^{d}-30$ strain resulting in, for instance, compact growth. Because of the role polyols have in maintaining the osmotic balance (Beever \& Laracy, 1986; Van Laere, 1989), it is likely that the total internal concentration of these polyols remains constant for a given set of growth conditions. Polyols which are overproduced are therefore likely to be secreted, as was found with the $\operatorname{cre} A^{d}$-30 mutant, which had high extracellular levels of all polyols although the total internal polyol concentration was almost the same as that in the wild-type (Table 3).

The increased internal concentration of the metabolites
F1,6dP, 2PG, 3PG and PEP in the cre $A^{d}-30$ mutant (Table 2 ) is likely to result from the decreased activity of pyruvate kinase in the $\operatorname{cre} A^{d}-30$ mutant. The increased internal concentration of these metabolites might explain the formation of glycerol in the cre $A^{d}$-30 mutant. Reduced pyruvate kinase activity is also reflected by lower amounts of pyruvate and citrate in the mutant.

In yeast and fungi, glycolysis is controlled mainly at the level of phosphofructokinase and pyruvate kinase (for a review see, e.g. Gancedo \& Serrano, 1989). In A. niger, phosphofructokinase is also a key point in regulation of the glycolytic flux, which is activated by $\mathrm{NH}_{4}^{+}, \mathrm{AMP}$ and $\mathrm{F} 2,6 \mathrm{bP}$ and is inhibited by citrate and PEP (Habison et al., 1983; Arts et al., 1987). In the cre $A^{d}$-30 strain, phosphofructokinase activity was lower than in the wild-type strain. However, the concentration of F2,6bP was higher. It is possible that this increase compensates for the lower amount of phosphofructokinase, but it is not known whether in the cre $A^{d}$-30 strain this results in vivo in phosphofructokinase activity that is comparable with that in the wild-type strain.

Another control point of glycolysis is the first step, the phosphorylation of glucose by hexokinase. In yeast, a strong correlation was found between the catalytic activity of the gene $b x k 2$, which encodes the hexokinase isoenzyme P-II, and its capacity to mediate catabolite repression (Ma et al., 1989; Rose et al., 1991). Furthermore, Saccharomyces cerevisiae hexokinase activity was recently found to be strongly inhibited by trehalose 6phosphate (T6P) (Blázquez et al., 1993). In wild-type $A$. nidulans, more trehalose was detected than in the $\operatorname{cre} A^{d}-30$ strain, suggesting that the flux towards trehalose formation is higher in the wild-type or, more likely, that trehalose turnover is higher in the $\operatorname{cre} A^{d}$-30 strain. Recently it has been found that hexokinase in $A$. nidulans is also inhibited by T6P, suggesting similar regulation to that in S. cerevisiae (G. J. G. Ruijter \& J. Visser, unpublished results). Interestingly, in S. cerevisiae there are several putative binding sites for the carbon catabolite repressor protein MIG1 in the promoter region of $b x k 2$, but the $A$. nidulans equivalent of the latter gene has not been cloned yet.

In summary, the cre $A^{d}$-30 mutation is responsible for radical changes in $\mathrm{D}$-glucose degradation. These changes occur mainly at the level of those enzymes known to play a role in regulating glycolysis, namely hexokinase, phosphofructokinase and pyruvate kinase. The result of these changes is a higher flux through the side chains towards formation of polyols and a possible decreased flux through the tricarboxylic acid cycle due to lower activity of pyruvate kinase. It is not known, however, if the changes found in the different enzyme levels are a result of a direct or an indirect effect of the cre $A$ mutation. Polyol catabolism in $A$. nidulans is known to be affected by carbon catabolite repression as shown for the utilization of glycerol (Hondmann \& Visser, 1992) and erythritol (Hondmann, 1994). We have insufficient knowledge of how carbon catabolite repression affects polyol biosynthesis (cf. Fig. 1). Although this study indicates the fructose-6-phosphate reductase gene to be a potential 
CREA target in mannitol biosynthesis, information about CREA-responsive steps in polyol biosynthesis at the C5, $\mathrm{C} 4$ and $\mathrm{C} 3$ level is not available. The overall conclusion from our data is that polyol turnover will be higher in cre $A^{d}$-30.

The changes found did not disturb the energy charge in the $\operatorname{cre} A^{d}-30$ mutant which is comparable to that of the wild-type. Besides these effects found in D-glucose degradation, it is likely that more enzyme systems will be affected by the $\operatorname{cre} A$ mutation, which should be taken into account in interpreting studies of gene regulation using $\operatorname{cre} A$ mutants. The accumulation of glycerol in the $\operatorname{cre} A^{d_{-}}$ 30 mutant might be the prime reason, for instance, why its alc $A$ and ald $A$ expression is high (Mathieu \& Felenbok, 1994), although there would be no further physiological consequences in the absence of a suitable substrate.

\section{ACKNOWLEDGEMENTS}

This project was financed by a grant from the Programme Committee for Agricultural Biotechnology (PcLB) (grant no. 8469) and by the European Commission to J.V. (BRIDGE grant no. CT90-0169 and BIOTECH CT93-0174). The authors thank Professor H. N. Arst, Jr, for critical reading of the manuscript.

\section{REFERENCES}

Arst, H. N., Jr \& Cove, D. J. (1973). Nitrogen metabolite repression in Aspergillus nidulans. Mol \& Gen Genet 126, 111-141.

Arst, H. N., Jr \& MacDonald, D. W. (1975). A gene cluster of Aspergillus nidulans with an internally located cis-acting regulatory region. Nature 254, 26-31.

Arst, H. N., Jr \& Bailey, C. R. (1977). The regulation of carbon metabolism in Aspergillus nidulans. In Genetics and Pbysiology of Aspergillus, pp. 131-146. Edited by J. E. Smith \& J. A. Pateman. London: Academic Press.

Arst, H. N., Jr, Tollervey, D., Dowzer, C. E. A. \& Kelly, J. M. (1990). An inversion truncating the cre $A$ gene of $A$ spergillus nidulans results in carbon catabolite derepression. Mol Microbiol 4, 851-854.

Arts, E., Kubicek, C. P. \& Rohr, M. (1987). Regulation of phosphofructokinase from Aspergillus niger: effect of fructose-2,6bisphosphate on the action of citrate, ammonium ions and AMP. $J$ Gen Microbiol 133, 1195-1199.

Bailey, C. \& Arst, H. N., Jr (1975). Carbon catabolite repression in Aspergillus nidulans. Eur J Biochem 51, 573-577.

Beever, R. E. \& Laracy, E. P. (1986). Osmotic adjustment in the filamentous fungus Aspergillus nidulans. J Bacteriol 168, 1358-1365.

Bergmeyer, H. U. (1985a). Methods of Enzymatic Analysis, vol. 6. Deerfield Beach, FL: VCH Publishers.

Bergmeyer, H. U. (1985b). Methods of Enzymatic Analysis, vol. 7. Deerfield Beach, FL: VCH Publishers.

Blázquez, M. A., Lagunas, R., Gancedo, C. \& Gancedo, J. M. (1993). Trehalose-6-phosphate, a new regulator of yeast glycolysis that inhibits hexokinases. FEBS Lett 329, 51-54.

Cubero, B. \& Scazzocchio, C. (1994). Two different, adjacent and divergent zinc finger binding sites are necessary for CREAmediated carbon catabolite repression in the proline gene cluster of Aspergillus nidulans. EMBO J 13, 407-415.
Dowzer, C. E. A. \& Kelly, J. M. (1989). Cloning of the creA gene from Aspergillus nidulans: a gene involved in carbon catabolite repression. Curr Genet 15, 457-459.

Dowzer, C. E. A. \& Kelly, J. M. (1991). Analysis of the cre $A$ gene, a regulator of carbon catabolite repression in Aspergillus nidulans. Mol Cell Biol 11, 5701-5709.

Gancedo, C. \& Serrano, R. (1989). Energy-yielding metabolism. In The Yeasts, vol. 3, pp. 205-259. Edited by A. H. Rose \& J. S. Harrison. New York: Academic Press.

Habison, A., Kubicek, C. P. \& Röhr, M. (1983). Partial purification and regulatory properties of phosphofructokinase from Aspergillus niger. Biochem J 209, 669-676.

Hondmann, D. H. A. (1994). Purification, properties and pbysiological significance of $\mathrm{NAD}{ }^{+}$-dependent erythritol debydrogenase from Aspergillus nidulans, pp. 105-117. $\mathrm{PhD}$ thesis, Wageningen Agricultural University, The Netherlands.

Hondmann, D. H. A. \& Visser, J. (1992). Selection and characterization of Aspergillus nidulans mutants involved in regulation of glycerol metabolism. $J$ Gen Microbiol 138, 809-815.

de Koning, W. \& van Dam, K. (1992). A method for the determination of changes in glycolytic metabolites in yeast on a subsecond time scale using extraction at neutral pH. Anal Biochem 204, 118-123.

Kulmburg, P., Mathieu, M., Dowzer, C., Kelly, J. \& Felenbok, B. (1993). Specific binding sites in the alc $\mathrm{R}$ and alc $A$ promotors of the ethanol regulon for the CREA repressor mediating carbon catabolite repression in Aspergillus nidulans. Mol Microbiol 7, 847-857.

Ma, H., Bloom, L. M., Zhu, Z. M., Walsh, C. T. \& Botstein, D. (1989). Isolation and characterization of mutations in the Hxk2 gene of Saccharomyces cerevisiae. Mol Cell Biol 12, 5630-5642.

Mathieu, M. \& Felenbok, B. (1994). The Aspergillus nidulans CREA protein mediates glucose repression of the ethanol regulon at various levels through competition with the ALCR-specific transactivator. EMBO J 13, 4022-4027.

Pontecorvo, G., Roper, J. A., Hemmons, L. M., Macdonald, K. D. \& Button, A. W. J. (1953). The genetics of Aspergillus nidulans. $A d v$ Genet 5, 141-238.

Rose, M., Albig, W. \& Entian, K. D. (1991). Glucose repression in Saccharomyces cerevisiae is directly associated with hexose phosphorylation by hexokinases PI and PII. Eur J Biochem 3, 511-18.

Van Laere, A. (1989). Trehalose, reserve and/or stress metabolite? FEMS Microbiol Rev 63, 201-210.

Van Schaftingen, E., Lederer, B., Bartrons, R. \& Hers, H. G. (1982). A kinetic study of pyrophosphate: fructose-6-phosphate transferase from potato tubers. Eur J Biochem 129, 191-195.

van der Veen, P., Flipphi, M. J. A., Voragen, A. G. J. \& Visser, J. (1991). Induction, purification and characterisation of arabinases produced by Aspergillus niger. Arch Microbiol 157, 23-28.

van der Veen, P., Arst, H. N., Jr, Flipphi, M. J. A. \& Visser, J. (1994). Extracellular arabinases in Aspergillus nidulans: The effect of different cre mutations on enzyme levels. Arch Microbiol 162, 433-440.

Witteveen, C. F. B. (1993). Gluconate formation and polyol metabolism in Aspergillus niger, pp. 107-122. PhD thesis, Wageningen Agricultural University, The Netherlands.

Witteveen, C. F. B., Busink, R., van de Vondervoort, P., Dijkema, C., Swart, K. \& Visser, J. (1989). L-Arabinose and D-xylose catabolism in Aspergillus niger. J Gen Microbiol 135, 2163-2171.

Received 7 December 1994; revised 17 April 1995; accepted 9 May 1995. 